\title{
SECTION FRANCAISE
}

Par suite des circonstances que nous n' avons pu prévoir, nous ne sommos pas encore à même de donner dans notre revue à la France la place à laquelle elle pourrait prétendre, vu sa tradition culturelle séculaire.

Déjà avant la guerre nous étions en communication avec le Centre d'Etude des Problèmes Humains (le G.E.P.H.) à Paris, qui, comme nous, avait réuni autour de lui un certain nombre de spécialistes adonnés à l'etude des "sciences de l'homme".

Un des problèmes que nous nous étions proposé d'envisager ensemble était au fond celui des ,conditions d'équilibre intérieur des individus et des conditions d'équilibre extérieur des individus entre eux". Faute d'esprits rigoureusement encyclopédiques ces problèmes ne peuvent, à l'avis des Centres, être étudiés que collectivement, par des équipes de spécialistes de toutes les facultés et par des forces dont la composition engendre l'équilibre étudié. Cette méthode collective de recherches semble devoir s'imposer de plus en plus à la science moderne, tant pour la recherche même que pour la documentation.

Nous publions ici le texte d'une conférence qu'un représentant de notre Rédaction aurait faite à Pontigny en 1938 et qui a paru également dans "Humanisme Economique", organe da C.E.P.H.

\section{$* * *$}

\section{POUR UNE MEILLEURE COMPREHENSION MUTUELLE}

L'incompréhension qui sépare les hommes et rend souvent difficile leur collaboration, est dûe en partie à l'imprécision de la langue, outil ancien qui n'a pas toujours l'acuité nécessaire pour servir efficacement les subtilités de la pensée scientifique moderne.

De bons esprits se sont inquiétés de cet état de choses, et ont aperçu dans le Centre d'Etude des Problèmes Humains une tribune à la diffusion de leurs préoccupations.

C'est ainsi que la communication suivante nous a été transmise. Destinée dans l'esprit des auteurs à être présentée à Pontigny le ler octobre, elle n'a pu trouver place dans un programme que les événements ont obligé à resteindre aux seules questions de politique internationale.

Nous attachons trop de prix à tout ce qui peut contribuer à rapprocher les hommes, à l'intérieur et par-dessus les frontières. pour ne. pas offrir avec plaisir à nos amis néerlandais l'hospitalité de notre Revue.

La Direction du C.E.P.H. 